\title{
Effect of Thermal Stress on Dairy Animal
}

\author{
Ranjan Kumar ${ }^{1 *}$, RK Tiwari ${ }^{1}$, Brajesh Shahi' ${ }^{2}$ and SK Saha ${ }^{2}$ \\ ${ }^{1}$ Dr Rajendra Prasad Central Agricultural University, India \\ ${ }^{2}$ Indian Veterinary Research Institute, India
}

Received: 畊 April 09, 2018; Published: 此 April 17, 2018

*Corresponding author: Ranjan Kumar, Dr Rajendra Prasad Central Agricultural University, Pusa, Bihar, India

\section{Introduction}

Thermal stress or heat stress can be defined as the sum of external forces to a homoeothermic animal that acts to displace body temperature from the resting state. Such a stress can disrupt the physiologic and productive performance of an animal. The increase in body temperature caused by heat stress has direct, adverse consequences on cellular functions. The livestock's by homeotherms to the stabilize body temperature within fairly narrow limits is essential to control biochemical reactions and physiological processes within normal metabolism [1]. In order to maintain homeothermy, an animal must be in thermal equilibrium with its environment, which includes radiation, air temperature, air movement and humidity. Body temperature is regulated by modulation of metabolic heat production and heat loss from the body through sensible and insensible means. The deviation in ambient temperature below or above the thermoneutral zone (5250C) causes thermal stress to the animals. Cattle and buffaloes can maintain their physiological processes within the normal limits in an ambient temperature of 5-250C [2]. Hot humid season is more stressful to livestock species compared to hot dry season mainly due to lower evaporate and heat loss from the animal body. The evaporate heat loss takes place through respiratory tract (panting) and skin surface (sweating) in animals. Nonevaporate heat exchange occurs through conduction, convection and radiation and depends on temperature gradients between animal and surrounding environment and vice versa. Heat stress causes behavioral and metabolic changes and thereby reduces feed intake and metabolic activity and ultimately decline in animal's productivity.

\section{Physiological effects of heat stress}

Numerous physiological changes occurs in the digestive system, acid-base chemistry, and blood hormones during heat stress that leads to reduced nutrient intake, but many changes occurs as a result of strain in the animals. The temperature sensitive neurons which are located throughout the animal's body and send information to the hypothalamus, which invokes numerous physiological, anatomical or behavioral changes in the attempt to maintain heat balance. During heat stress cows exhibit reduces feed intake, decreased activity, seek, shade and wind, increase respiratory rate, and increase both peripheral blood flow and sweating. These responses have deleterious effect on both production and physiologic status of the animal. Cows that were fed ad libitum in a thermal comfort environment, fed ad libitum in a thermal stress environment, or feed restricted intake in a thermal comfort environment had similar milk yields for both restricted intake and thermal stress treatments, and mammary blood flow tended to be lower compared with ad libitum feed cows in thermal comfort, suggesting blood flow was responsive to level of dry matter intake (DMI). The negative effects of heat stress on milk production is due to decreased nutrient intake and decreased nutrient uptake by the portal drained viscera of the cow. Which leads to blood flow shifted to peripheral tissues for cooling purposes may alter nutrient metabolism and contribute to lower milk yield during heat weather.

\section{Metabolic effect}

Heat stress causes reduced metabolism in cattle leads to reduced thyroid hormone secretion and gut motility, resulting in increased gut fill. Higher blood plasma content of epinephrine and norephinephrine with high ambient temperatures is an indicator of stress response and reduces rate of passage in the digestive tract. Slower passage rate can lead to greater gut fill and limited intake but improved digestibility because of greater residence time in the gut.

\section{Effect on electrolytes and minerals}

With increased respiration during heat stress, the expiration of $\mathrm{CO}_{2}$ exceeds the rate of its formation in the body. The partial pressure of $\mathrm{CO}_{2}$ of blood declines, creating a deficit of blood carbonic acid and resulting in respiratory alkalosis. This leads to elevated blood and urine $\mathrm{PH}$ and net acid excretion rate. The cow compensates 
for high blood $\mathrm{PH}$ by excreting $\mathrm{HCO}_{3}$ ions into the urine. Alkalemia depresses the rate of renal secretion of hydrogen ions, but increases the excretion of filtered $\mathrm{HCO}_{3}$, which subsequently leads to reduction in blood $\mathrm{HCO}_{3}$ concentration. Metabolic acidosis may occur if available Na in these regions is reduced, possibly increasing chloride absorption, draining $\mathrm{HCO}_{3}$ and increasing $\mathrm{Cl}$ in the plasma. Metabolic alkalosis may result from a reciprocal process.

During hot weather, declining DMI and high lactation demand requires increased dietary mineral concentration. However, alterations in mineral metabolism also effect the electrolyte status of the cow during hot weather. The primary cation in bovine sweat is potassium $(\mathrm{K})$ and sharp increases in the secretion of $K$ through sweat occur during hot climatic conditions. The absorption of macro-minerals, including $\mathrm{Ca}, \mathrm{P}$, and $\mathrm{K}$, declined during hot temperatures and also the trace element requirements may increase with elevated environment temperature. Lactating cows subjected to hot climatic conditions and supplemented with $\mathrm{K}$ well above minimum NRC recommendations (NRC, 1989) responded with greater milk yields.

Electrolyte concentration particularly, $\mathrm{Na}$ and $\mathrm{K}$ are reduced in rumen fluid of heat stressed dairy cattle because of increased loss of $\mathrm{Na}$ in urine and $\mathrm{K}$ in the sweat. High environmental temperature may affect the rumen microorganisms that synthesize B- vitamins, amino acids and fatty acids. It is suggested that rumination is suppressed during dehydration and heat stress. The lower ruminal PH because of high conc. of lactic acid in heat stressed animal might be involved in inhibiting rumen motility. Because of inhibition of reticulo-rumen motility reduced conc. of (volatile fatty acids) VFA in rumen of heat stressed cattle.

\section{Effect on Reproductive system}

Heat stress reduces the length and intensity of estrus through the involvement of hypothalamus-hypophyseal-gonodal axis. Heat stress can cause an increase cortisol secretion and block estradiol induced sexual behavior. There is an altered secrection of LH and FSH at the hypophyseal level and steroids (androstenedione, progesterone and estradiol) at ovarian level in heat stressed animals. However in certain experiments it has been observed that circulatory cortisol concentration were transitatory during heat stress animal. The major effect of heat stress are, decreases the expression of estrus behavior, because of the physical lethargy produced by heat stress.

Reduced physical activity its probably an adaptive response that limits heat production. The uterine and oviductal tissues may be compromised during heat stress for several reasons first, heat stress lead to a redistribution of blood from viscera organs to peripheral this result decreased perfusion of nutrients and hormone could compromise endometrial functions. Recent experiments suggest that heat stress can cause increase peripheral concentration of estradiol-17 $\beta$ between 1 and 4 days of estrus cycle and 11-12 of the cycle.

There are variation of hormonal responses to heat stress probably reflects the fact that ovarian steroid concentrations are depended not only rate to secretion from ovarian tissue but also On rate of vascular perfusion of ovary or on metabolism in liver's other organ. During heat stress the action of steroid hormone on reproductive tract become reduced because of increased synthesis of heat shock protein. Heat shock can lead to increased synthesis of HSP70 and heat shock protein 90 in endometrium. These protein are part of the complex of protein associating with progesterone and estrogen receptor. Increased synthesis of heats and protein might alter assembly, transport or binding of steroid receptor.

\section{Conclusion}

The effect of heat stress includes reduction in feed intake and estrus symptoms, suppression of rumination, alteration of reduction of $\mathrm{Na}$ and $\mathrm{K}$ level in rumen fluid of dairy animals, because of increased loss of $\mathrm{Na}$ in urine and $\mathrm{K}$ in the sweat. High environmental temperature may also affect the rumen microorganisms that synthesize B- vitamins, amino acids and fatty acids. Extensive research efforts made in past decades to mitigate the effect of heat stress in dairy animals. Generally, there are three fundamental managemental practices namely physical protection, genetic development and nutritional management have been proposed to alleviate the extent and severity of thermal stress in animals. Alteration of the dairy animal's environment, genetic selection of more heat tolerance breed of heifers, nutritional management like grazing, balancing of ration, supplementation of bypass protein and bypass fat, antioxidant, use of neutraceuticals have been directed to reduce effect of heat stress in dairy animals and to improve the efficiency.

\section{References}

1. (1989) National Research Council Effect of Environment on Nutrient Requirement of Domestic Animals. National Academy Press Washington DC, USA.

2. McDowell RE, Hooven NW, Camoens JK (1976) Effects of climate on performance of Holsteins in first lactation. J Dairy Sci 59(5): 965-971. 
This work is licensed under Creative Commons Attribution 4.0 License

Submission Link:

Submit Article

DOI: $10.32474 / C D V S .2018 .01 .000105$

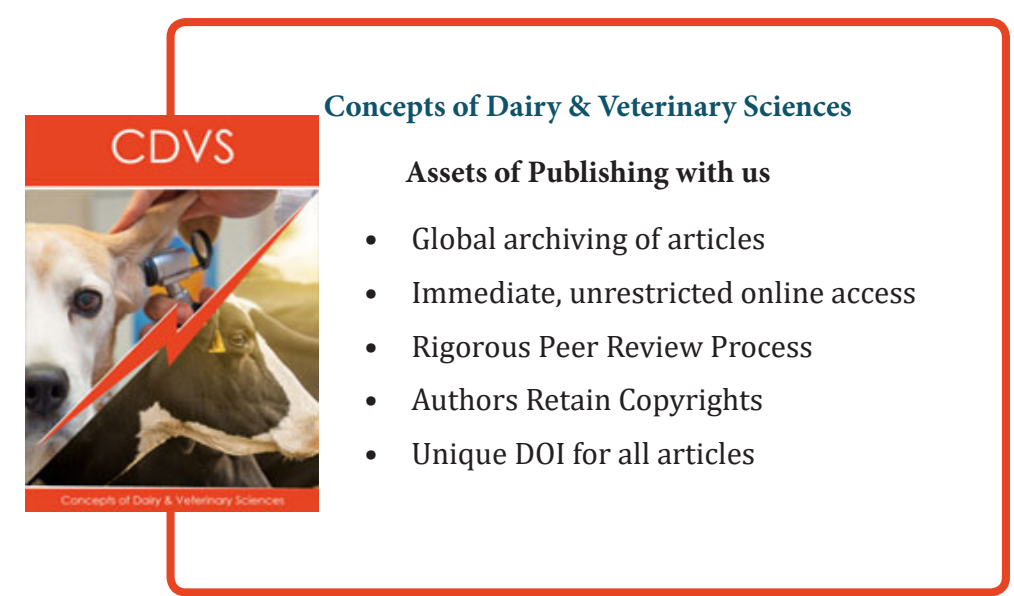

